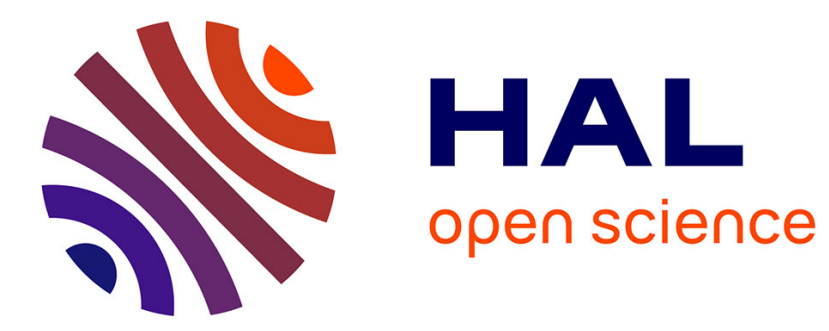

\title{
Ultrafast Maximum-Quantum NMR Spectroscopy for the Analysis of Aromatic Mixtures
}

Maria Grazia Concilio, Corentin Jacquemmoz, Dina Boyarskaya, Géraldine Masson, Jean-Nicolas Dumez

\section{- To cite this version:}

Maria Grazia Concilio, Corentin Jacquemmoz, Dina Boyarskaya, Géraldine Masson, Jean-Nicolas Dumez. Ultrafast Maximum-Quantum NMR Spectroscopy for the Analysis of Aromatic Mixtures. ChemPhysChem, 2018, 19 (23), pp.3310-3317. 10.1002/cphc.201800667 . hal-02324695

\section{HAL Id: hal-02324695 \\ https://hal.science/hal-02324695}

Submitted on 19 Jan 2021

HAL is a multi-disciplinary open access archive for the deposit and dissemination of scientific research documents, whether they are published or not. The documents may come from teaching and research institutions in France or abroad, or from public or private research centers.
L'archive ouverte pluridisciplinaire HAL, est destinée au dépôt et à la diffusion de documents scientifiques de niveau recherche, publiés ou non, émanant des établissements d'enseignement et de recherche français ou étrangers, des laboratoires publics ou privés. 


\title{
Ultrafast maximum quantum NMR spectroscopy for the analysis of aromatic mixtures
}

\author{
Maria Grazia Concilio, Corentin Jacquemmoz, Dina Boyarskaya, Géraldine Masson and Jean-Nicolas \\ Dumez*
}

\begin{abstract}
Maximum-quantum (MaxQ) NMR experiments have been introduced to overcome issues related to peak overlap and high spectral density in the NMR spectra of aromatic mixtures. In MaxQ NMR, spin systems are separated on the basis of the highest-quantum coherence that they can form. MaxQ experiments are however time consuming and methods have been introduced to accelerate them. In this article, we demonstrate the ultrafast, single-scan acquisition of MaxQ NMR spectra, using spatial encoding of the multiple-quantum dimension. So far, the spatial encoding methodology has been applied only for the encoding of up to double-quantum coherences, and here we show that it can be extended to higher coherence orders, to yield a massive reduction of the acquisition time of multi-quantum spectra of aromatic mixtures, and also to monitor chemical reactions.
\end{abstract}

\section{Introduction}

Nuclear magnetic resonance (NMR) spectroscopy is a powerful tool for the analysis of complex mixtures of small molecules in solution. Advanced NMR methods, often based on sophisticated multidimensional (ND) schemes [1-3], have been developed to overcome the issues of signal overlap and high spectral density, which limit the applicability of $1 D$ NMR for mixtures analysis. A particularly useful method in the case of aromatic compounds is maximum-quantum (MaxQ) NMR, based on multiple-quantum NMR [4-8]. MaxQ NMR relies on the encoding of multiple-quantum coherences. Specifically, when the highest-quantum coherence (the MaxQ) present in a given spin system is encoded, the corresponding peak in the indirect dimension reduces to a single line that resonates at a position given by the sum of the $1 \mathrm{Q}$ chemical shifts of the spins participating to the MaxQ. This simplifies drastically the multiple-quantum dimension, allowing a differentiated identification of the compounds in the mixture [9-12]. This technique has been successfully used to simplify crowded spectra of several kinds of mixtures. Other applications of multiple-quantum NMR include spin counting in MQ filtered COSY or ${ }^{13} \mathrm{C}$ detected INADEQUATE experiments [7, 13-15], and the identification of spin topologies [16] for spin state selection relying on heteronuclear couplings.

Multidimensional NMR methods such as MaxQ NMR are however, due to the presence of one or several indirect evolution dimensions, more time consuming and unsuitable for

Institut de Chimie des Substances Naturelles, CNRS UPR2301, Univ.Paris Sud, Université Paris-Saclay. 1, avenue de la Terrasse, 91198 Gif-sur-Yvette,France.

* Corresponding author. jeannicolas.dumez@cnrs.fr (J.-N. Dumez) Supporting information for this article is given via a link at the end of the document. studies of dynamical processes and/or unstable samples, which require appropriate time resolution. Several strategies have been developed to overcome the time limitation of multidimensional NMR. Some of these, are based on a reduced inter-scan delay that can be optimized together with the radiofrequency (RF) excitation pulse angles [17, 18], on non-uniform sampling (NUS) of the multidimensional data space coupled with non-Fourier signal processing methods [19-21], on Hadamard encoding [22 - 23], and on covariance analysis [2425]. Examples of applications of fast $2 D$ NMR for reaction monitoring include the kinetic study of an organic reaction [2627], the analysis of bacterial metabolism in situ [28] the monitoring of a phosphorylation biochemical reaction [29]. NUS, in conjunction with Recursive Multi-Dimensional Decomposition (R-MDD) data processing, has been combined with MaxQ NMR for an accelerated acquisition [11]. The most significant acceleration of 2D NMR experiments is obtained with the concept of "ultrafast" 2D NMR, which relies on spatial encoding of the indirect dimension and yields a complete data set in a single scan. UF 2D NMR has been used for a large number of applications [30], including real-time reaction monitoring [31-33], and so far it has been applied so far only for the encoding of coherence orders of up to two. [34-35]

In this article, we demonstrate the ultrafast (UF), singlescan acquisition of multiple-quantum NMR spectra, using spatial encoding of the multiple-quantum coherences of coherence orders of up to five. We show how the resulting method can be used for the maximum-quantum analysis of a model mixture of aromatic compounds, and also for reaction monitoring, with significantly reduced experiment durations. The analysis is supported by numerical spin simulations, which also help to assign the MaxQ spectra. The resulting methods will be useful for the analysis of mixtures containing a variety of structurally similar aromatic compounds, such as polyphenol, flavanols and secoiridoids [12]. 


\section{Theory}

The UF2DNMR approach was designed by Frydman and co-workers [36-39], and has already been applied to a variety of NMR pulse sequences, such as COSY, TOCSY and HSQC for the study of complex mixtures with significantly reduced experimental times. [30] The NMR pulse sequences for ultrafast multiple-quantum (UFMQ) NMR spectroscopy, used in this work, are shown in Fig. 1.

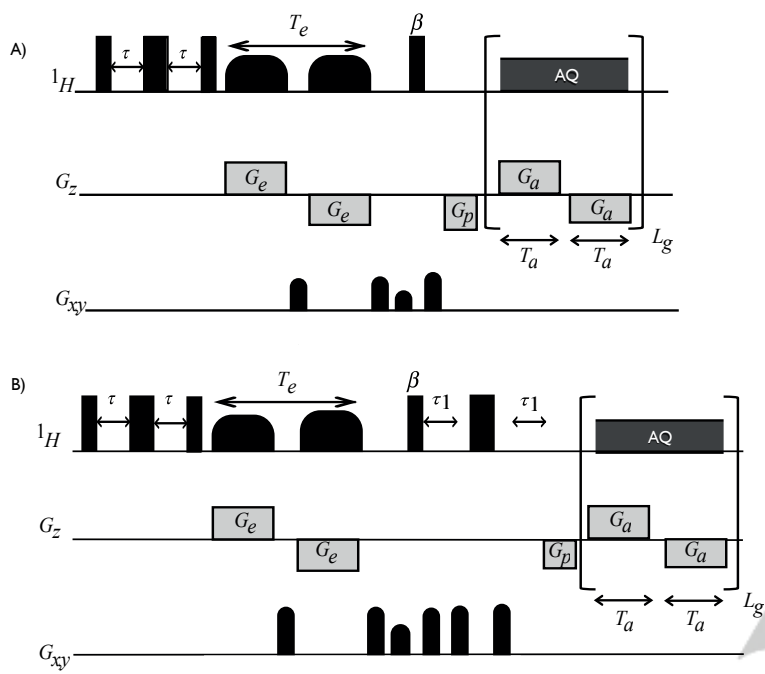

Fig. 1. Pulse sequences for (A) UF MQ without refocusing period. (B) UF $M Q$ with refocusing period.

The general setting up of UF2DNMR pulse sequences consists in the replacement of the evolution period $\left(t_{1}\right)$ of a conventional pulse sequence by a spatial encoding scheme, based on the combination of bipolar gradients (encoding gradients, $G_{e}$ ) and pulses whose frequencies are swept linearly in time - also known as chirp pulses. As the conventional MQ NMR sequences, widely described in the literature [4-8], the UF sequences contain a spin-echo block (Fig. 1), in which the second $90^{\circ}$ pulse is used to generate $M Q$ coherences, of even order when applied on the $x$-axis and of odd order when applied on the $y$-axis (assuming a first $90^{\circ}$ pulse along $x$ ). The mixing pulse, indicated as flip angle $\beta$, is used to convert $\mathrm{MQ}$ coherences into single quantum. Coherence selection gradients that flank the mixing pulse are used to select the coherence order during the evolution period. Sequence $A$ and $B$ shown in Fig. 1 differ by the use of a refocusing block used to add additional time to let anti-phase terms evolve into in-phase terms, which can lead to an improved signal-to-noise ratio, as explained below.

The difference between UF and conventional NMR consists in the spatial partitioning of the sample into a set of slices in which independent experiments are performed in parallel instead of sequentially. This approach creates a position-dependent evolution time of the form, $t_{1}(z)=C z$, where $C$ is a constant that depends on the pulse sequence and $z$ is the position along the longitudinal axis. UF experiments described in this work are performed using a constant time (CT) scheme [31], to let spins spend the same amount of time in the transverse plane regardless of their position in the sample. The purpose of the gradients, applied along the z-axis, is to create a position-dependent off-resonance frequency, while the purpose of the first chirp is to rotate spins into the $x$-axis sequentially in space and time. Consider a multiple-quantum coherence of the form $\prod_{i=1}^{p} L_{i}^{+}$, the overall phase shift acquired by the $\mathrm{MQ}$, at the end of the first chirp pulse, is given by the sum of the phase shift for each single quantum coherence, and can be described by Eq. 1 .

$$
\varphi^{(\pi)}(z)=-\varphi_{0}(z)+\left(p \frac{\left(\gamma G_{e}\right)^{2}}{R}\right) z^{2}+\frac{2 \gamma G_{e} T^{(\pi)} \sum_{i=1}^{p} \Omega_{i}}{B W} z+Z_{0}
$$

where $\varphi^{(\pi)}(z)=0$ is the spin phase before the application of the chirp pulse, $p$ is the coherence order, $R=B W / T_{e}$ is the sweep rate, $\Omega_{i}$ is the chemical shift offsets involved. $Z_{0}$ is a $z$-independent term that is not considered here (all the spectra are processed in magnitude mode). The phase acquired by the $M Q$ contains a desirable linear part in $z$, but also unwanted quadratic terms proportional to $z^{2}$. In order to eliminate the quadratic term, a second chirp is applied with identical duration, sweep rate and gradient amplitude but with opposite sign of the gradient. After the second chirp, the phase shifts of the MQ is given by Eq. 2:

$$
\varphi^{(\pi-\pi)}(z)=\varphi_{0}(z)-\frac{4 \gamma G_{e} T^{(\pi)} \sum_{i=1}^{p} \Omega_{i}}{B W} z
$$

The expression in Eq. 2 comes from the second term of Eq. 1, it has a prefactor of four rather than two, as in Eq. 1, and is negative due to the presence of the second chirp pulse. Eq. 2 can be rewritten, normalizing to the sample length such that the coefficient of the quadratic phase in Eq. 1 becomes equal to the product of the sweep rate and the duration of the chirp that is proportional to its bandwidth. Therefore, writing the sample length as: $L_{z}=B W / \gamma G_{e}$ and then incorporating all the terms in a constant equal to: $C=4 T_{e} / L_{z}$, Eq. 2 can be reduced to Eq. 3:

$$
\varphi^{(\pi-\pi)}(z)=\varphi_{0}(z)-C z \sum_{i=1}^{p} \Omega_{i}
$$


Eq. 3 has the desired form, which is linear in $z$ and in the sum of the chemical shifts, with a proportionality constant $C$ that depends on the sequence parameters. The quadratic and linear phase shifts acquired by the $M Q$ coherences after the first chirp (given by Eq. 1) and after spatial encoding block (given by Eq. 3) were verified using spin dynamics simulations performed using Fokker-Planck theory in the Spinach software. [40, 41] As expected, a good agreement between analytical and numerical phase shifts was observed (Fig. S1 in ESI).

After the spatial encoding block, the phase of the $M Q$ coherence can be described by a helix around the z-axis, with contributions from different $\mathrm{z}$ values interfering destructively. With the mixing pulse, this positiondependent phase is transferred to observable singlequantum coherence. The application of a readout gradient then results in the formation of echoes, at a position that reflects the initial helical winding, and hence the encoded multiple-quantum chemical-shift. In order to unfold both the indirect (multiple-quantum) and direct (single-quantum) dimensions, MRI-inspired echo-planar spectroscopic imaging (EPSI) acquisition is used, based on a train of bipolar gradients $\left(G_{a}\right)$ with identical amplitude and duration repeated a number of times, $L_{g}$. A pre-phasing gradient, $G_{p}$, is used before acquisition to shift the spectrum in the $M Q$ dimension. The signal is then rearranged into a $2 \mathrm{D}$ matrix, and Fourier transformed along the direct dimension before magnitude calculation.

\section{Experimental and theoretical section}

\section{Sample preparation}

The model aromatic mixture (Fig. 2) studied in this work was prepared by dissolving six compounds: caffeic, ferulic and vanillic acids (MaxQ equal to 3 ) naphthalene and anthracene (MaxQ equal to 4) and phenol (MaxQ equal to 5 ) in DMSO-d 6 , to make a solution about $150 \mathrm{mM}$ of each compound.

UF MQ NMR was also used for reaction monitoring, with the example of a inverse electron-demand aza-DielsAlder reaction, reported by Masson and co-workers [42]. This concerns the reaction of the $\mathrm{N}$-phenyl imine, with (E)benzyl (penta-1,3-dien-1-yl)carbamate as dienophile, catalyzed by a racemic BINOL-phosphoric acid, affording the corresponding tetrahydroquinoline. The reaction was performed by adding benzyl N-[(1E)-prop-1-en-1-yl]carbamate (the dienophile, $39.33 \mathrm{mg}, 0.21 \mathrm{mmol}, 1.05$ equivalent) and a catalyst ( $1.98 \mathrm{mg}, 5 \mu \mathrm{mol}, 2.8 \% \mathrm{~mol})$ to a solution of $\mathrm{N}$-benzylideneaniline (imine, $36.61 \mathrm{mg}, 0.20$ mmol, 1.0 equivalent) in $0.5 \mathrm{~mL}$ of $\mathrm{CDCl}_{3}$, at room temperature. The mixture was then stirred for few seconds and then poured into an NMR tube before reaction monitoring.

\section{NMR spectroscopy}

Experiments on the model mixture were performed at a nominal temperature of $298 \mathrm{~K}$ using a Bruker $600 \mathrm{MHz}$ NMR spectrometer operating at a ${ }^{1} \mathrm{H}$ Larmor frequency of 600.13 MHz (AVANCE III console) equipped with a roomtemperature triple-axis gradient high-resolution probe (TXI) capable of generating $z$ gradients fields up to $65 \mathrm{G} / \mathrm{cm}$ and orthogonal gradients up to $50 \mathrm{G} / \mathrm{cm}$.

For the UF experiments, the amplitude of the encoding gradients was set equal to $0.78 \mathrm{G} / \mathrm{cm}$ in the $U F 3 Q$ experiments, equal to $0.65 \mathrm{G} / \mathrm{cm}$ in the UF $4 Q$ experiments and equal to $0.26 \mathrm{G} / \mathrm{cm}$ in the UF $5 \mathrm{Q}$ experiments, with a chirp pulse of $12 \mathrm{kHz}$ sweep width and duration $T_{e} / 2$, was kept constant and equal to $15 \mathrm{~ms}$. The amplitude of the encoding gradients was decreased in order to adapt the spectral width in the indirect dimension. The amplitude of the acquisition gradients was set equal to $26 \mathrm{G} / \mathrm{cm}$ in all the UF experiments, their duration was set equal to $428 \mu \mathrm{s}$, and the number of loops was set equal to 80 , resulting in an acquisition time of $72 \mathrm{~ms}$. The coherence selection gradients around the mixing pulse (with $\beta$ kept constant and equal to $90^{\circ}$ ) were determined in according to the $G_{2}=$ $p \times G_{1}$ rule, values of the $G_{1}$ and $G_{2}$ gradients were set in according to the coherence order to select. For these sequences, a more efficient de-phasing of unwanted coherences was obtained by placing pairs of orthogonal gradient pulses around the second chirp pulse and the refocusing pulse with length equal to $1 \mathrm{~ms}$ and amplitude equal to $10 \mathrm{G} / \mathrm{cm}$ and $35 \mathrm{G} / \mathrm{cm}$, respectively. For the UF experiments, the time that elapses between the excitation pulse and the end of acquisition is of less than 1 second. This duration is sufficient to encode the complete 2D information, and for single-scan experiments started at thermal equilibrium, it corresponds to the complete experimental duration. The total duration of the conventional $\mathrm{MQ}$ experiments was of about 10 minutes. For the UF experiments, the time that elapses between the excitation pulse and the end of acquisition is of less than 1 second. This duration is sufficient to encode the complete 2D information, and for single-scan experiments started at thermal equilibrium, it corresponds to the complete experimental duration. When multiple consecutive experiments were recorded (e.g. for build-up curves or reaction monitoring) a conservative relaxation delay of $10 \mathrm{~s}$ was used to avoid any hardware problem, resulting in a duration of about $10 \mathrm{~s}$ for each UF2DNMR experiment.

For both the UF and conventional experiments, the evolution time was also set equal to $30 \mathrm{~ms}$; the acquisition time was set equal to $72 \mathrm{~ms}$ in the UF experiments, and $200 \mathrm{~ms}$, in the conventional experiments. The processing of the conventional $\mathrm{MQ}$ experiment was performed using either the full FID or the first chunk of the FID with length equal to $72 \mathrm{~ms}$. All the experiments were acquired in one single scan per increment. 
A signal to noise (SNR) comparison between UF and conventional MQ NMR experiments, was performed on the model aromatic mixture with the same experimental conditions as those described above, but using the same total of scans for the conventional and UF experiment, resulting in the same total experiment time.

The cycloaddition reaction was monitored using both the same room-temperature triple-axis gradient probe (TXI) and a single axis cryoprobe CP TCl600 H-C/N, capable of generating $z$ gradients fields up to $53.5 \mathrm{G} / \mathrm{cm}$, using a Bruker NMR spectrometer operating at a ${ }^{1} \mathrm{H}$ Larmor frequency of $600.33 \mathrm{MHz}$ (AVANCE III console). Parameters in the experiment performed using the TXI, were set as the following: the amplitude of the encoding gradients was set equal $0.72 \mathrm{G} / \mathrm{cm}$ with a chirp pulse of 12 $\mathrm{kHz}$ sweep width and duration $T_{e} / 2$, kept constant and equal to $15 \mathrm{~ms}$. Parameters in the experiment performed using the single axis cryoprobe, were set as the following: the amplitude of the encoding gradients was set equal to $0.37 \mathrm{G} / \mathrm{cm}$ with a chirp pulse of $9 \mathrm{kHz}$ sweep width and duration $T_{e} / 2$, kept constant and equal to $15 \mathrm{~ms}$. The amplitude of the of the acquisition gradients was set equal to $27 \mathrm{G} / \mathrm{cm}$ in all the UF experiments, their duration was set equal to $428 \mu \mathrm{s}$. The number of loops was set equal to 80. The remaining parameters were the same as those used for the model mixture. The number of scans in the UF experiments, performed to monitor the Diels-Alder cycloaddition reaction, was set equal to 8 . UF experiments were acquired every 2 minutes; the total duration of each experiment was equal to 1 minute and 22 seconds.

Apodization of the conventional dimensions was performed using a sine bell function, while in the UF dimension Gaussian apodisation in the spatial domain was used. Simulated and experimental spectra are represented with a reduced chemical shift scale, for the indirect dimension, given by $\delta_{\max }^{R}=\sum_{i=0}^{p} \delta_{i 1 Q} / p$, where $\delta_{i 1 Q}$ are the $1 Q$ chemical shifts participating to that specific $M Q$ and $p$ is the coherence order of the MQ experiment.

\section{Simulations}

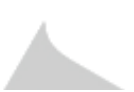

All the simulations were performed using the Spinach library, [40-41] the code developed for simulations and analysis will be publicly available from the version 2.3 and later.

\section{Results and Discussion}

The potential of UF 2D MQ NMR for the acquisition of MaxQ spectra of aromatic mixtures and for reaction monitoring is illustrated here. In the first example, UF MQ NMR is applied to a stable model mixture and comparison between UF and conventional MQ NMR spectra is carried out showing comparable signals are encoded in the two experiments. In the second example, a time series of UF 4Q NMR spectra are acquired at different stages of the Diels-Alder cycloaddition reaction.

\section{UF MaxQ NMR analysis of an aromatic mixture}

The chemical structures of the molecules of the aromatic mixture are shown in Fig. 2.

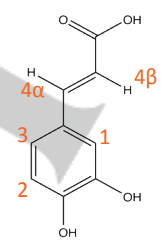

D

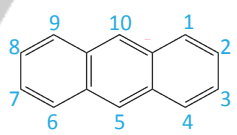<smiles>CCCCCC(=O)c1ccc(O)c(OC)c1</smiles>

$E$

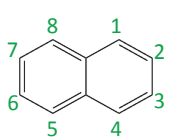<smiles>O=C(O)C=Cc1ccc(O)c(O)c1</smiles>

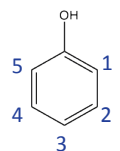

Fig. 2. Chemical structure of the six compounds used in this work, were: (A) caffeic acid, (B) ferulic acid, (C) vanillic acid, (D) anthracene $(E)$ naphthalene and $(F)$ phenol.

Fig. 3 shows the 1D NMR spectrum of the aromatic mixture studied in this work, showing the signal overlap that can be solved using 2D NMR.

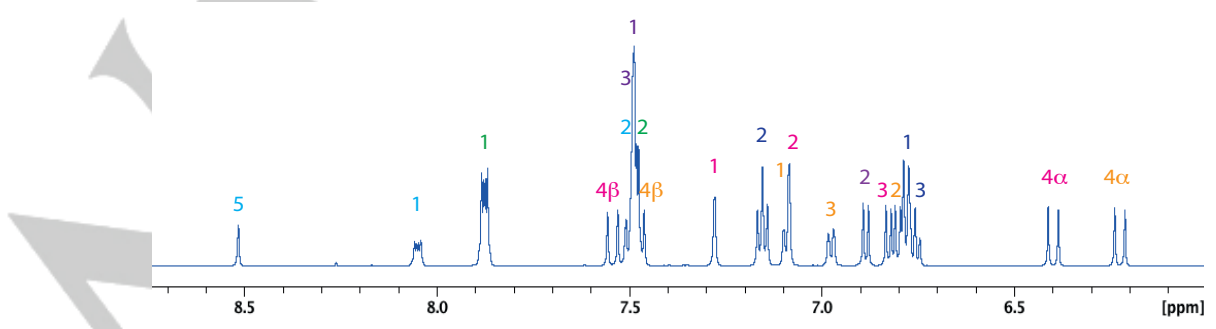

Fig. 3: 1D NMR spectrum of the aromatic mixture studied in this work, the colour code and numbering are referring to structures shown in Fig. 2. 
Figure 4 shows 2D NMR spectra acquired for the model mixture of aromatic compounds. The UF experiments were carried out with the sequence shown in Fig. $1 \mathrm{~B}$, and the conventional ones also included a spin-echo delay between mixing and detection, with values of the $\tau$ and $\tau^{\prime}$ delays chosen according to the strategy described below. Note that the conventional experiments are processed here with an acquisition time that matches that of the UF ones equal to $72 \mathrm{~ms}$, to compare the effect of the encoding mode only. Fig. S2.1 shows $3 Q, 4 Q$ and $5 \mathrm{Q}$ conventional NMR spectra processed with using the full FID with length equal to $200 \mathrm{~ms}$, characterized by better resolution.
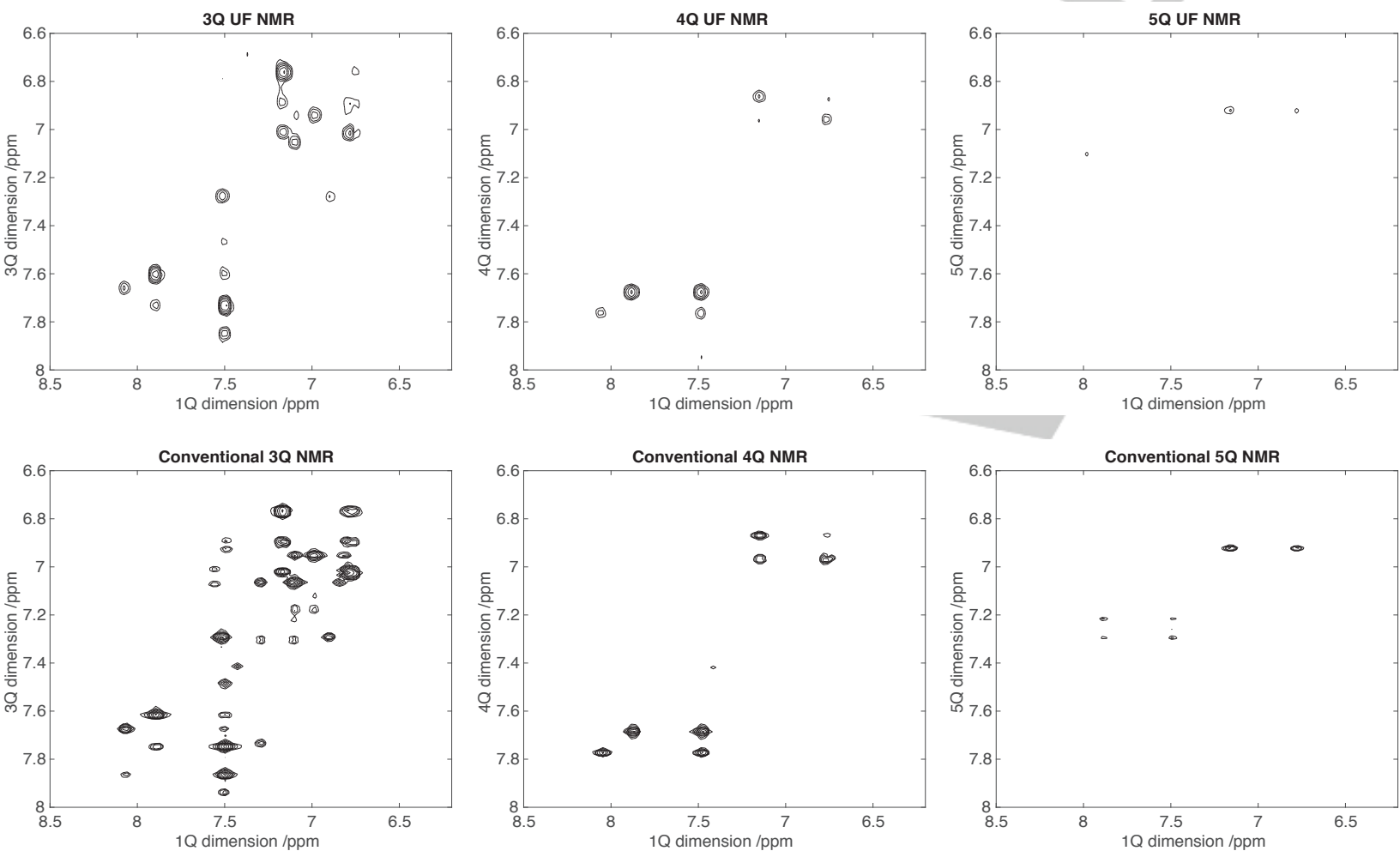

Fig. 4. Comparison between UF (top array) and conventional (bottom array) MQ NMR spectra of aromatic mixture studied in this work. Conventional spectra were processed using a contour level fifty times the root-mean-square ( $r m s)$ of the noise, while UF spectra were processed using a contour level three times the rms of the noise.

The full assignment of chemical shifts in both the $1 Q$ and $M Q$ dimensions for the correlations observed in the simulated and experimental spectra are reported in Tables S2.1- 2.3, and numerical simulations of the conventional MQ NMR experiments (Figs. S2.2.1 and S2.2.2) were performed to support the interpretation of the experimental spectra. A numerical code for the calculation of multiple-quantum chemical shifts was used for identification and assignment of the specific coherence orders, accelerating and simplifying the assignment for aromatic mixtures.

Most of the peaks observed in the conventional spectra were observed also in the UF spectra, confirming that multiplequantum coherences can be encoded spatially for coherence orders of up to 5 at least. In this case, spatial encoding reduces the experiment duration from several minutes to less than one second. Such speed however comes with a well-documented sensitivity penalty $[38,45]$, due to the increased acquisition bandwidth in UF2DNMR. In the UF $3 Q$ spectrum we did not observe $3 Q$ arising from weak couplings between protons within the ring and the acrylic functional groups of the caffeic and ferulic acids, represented by operators: $L_{3}^{+} L_{4 \alpha}^{+} L_{4 \beta}^{+}$
$(6.90$ ppm), $\quad L_{1}^{+} L_{4 \alpha}^{+} L_{4 \beta}^{+} \quad(6.93 \mathrm{ppm})$ and $L_{1}^{+} L_{3}^{+} L_{4 \beta}^{+} \quad(7.18 \mathrm{ppm})$ belonging to the caffeic acid, and operators: $L_{3}^{+} L_{4 \alpha}^{+} L_{4 \beta}^{+} \quad(7.02$ ppm), $\quad L_{1}^{+} L_{4 \alpha}^{+} L_{4 \beta}^{+}(7.08 \mathrm{ppm})$ and $L_{1}^{+} L_{3}^{+} L_{4 \beta}^{+} \quad(7.31 \mathrm{ppm})$, belonging to the ferulic acid. The sensitivity of the UF experiments can be improved to some extent by signal averaging. For example, averaging 16 scans gives a sensitivity increase of 4 , while maintaining a speed advantage over the conventional experiment; in the present case however this is not sufficient to recover the missing peaks. Improved sensitivity would also be obtained with the use of a cryogenically cooled probe, with the caveat that triple-axis gradients are less frequently available.

In the MaxQ approach, MQ NMR spectra are analyzed to identify correlations corresponding to complete spin systems. Three clear $3 \mathrm{Max} Q(\mathrm{~s})$, arising from caffeic, ferulic and vanillic acid, were observed in the $3 Q$ NMR spectra, as well as two $4 \mathrm{Max} Q(\mathrm{~s})$ from naphthalene and anthracene in the $4 \mathrm{Q}$ spectra, and one $5 \mathrm{MaxQ}$ from the phenol in the $5 \mathrm{Q}$ NMR spectra, the experimental chemical shifts of the $M Q$ in the indirect 
dimension, reported in the Table S2.2, matched those calculated, reported in the Table S2.3. However, not all the possible correlation peaks predicted were observed in the experimental data.

\section{Choice of experimental parameters}

The acquisition of the spectra shown in Fig. 4 and 7, obtained using the pulse sequence shown in Fig. 1B, required the optimization of the multiple-quantum build-up time $2 \tau$ and of a refocusing delay $2 \tau_{1}$ between the mixing pulse and acquisition. For UF2DNMR experiments carried out on high-resolution solution-state NMR hardware, the duration of the train of bipolar gradient is limited by the modest robustness of the gradient coil and amplifier. As a result, acquisition blocks of up to about 100 ms are typically used. If the acquisition is started just after the mixing pulse, the duration may not be enough for anti-phase single-quantum coherences to evolve into observable terms.
For this reason, a spin-echo block $(\tau-\pi-\tau)$ is inserted between the mixing pulse and the acquisition period.

The parameters $\tau$ and $\tau_{1}$ were optimised using the sequences $A$ (without the refocusing period) and $B$ (with the refocusing period) shown in Fig. 1. Fig. 5 shows data obtained from UF experiments performed using these sequences as well as equivalent conventional ones: the average behaviour of the peak volumes arising from $\mathrm{MQ}$ coherences belonging to phenol and naphtalene, is plotted as function of $\tau$ or $\tau_{1}$ (the full set of curves is shown in. Fig. S2.3). Sequence A was used to determine the $M Q$ build up times, by running a set of experiments with different $\tau$ delays. The value of $\tau$ corresponding to the highest peak volume, was then kept fixed in the sequence $B$, and a new set of experiments were performed with different $\tau_{1}$ delays in order to determine the time required to let the anti-phase terms to evolve into in-phase terms. Note that no $5 \mathrm{MaxQ}$ in the UF spectrum from the sequence without the refocusing block was observed.
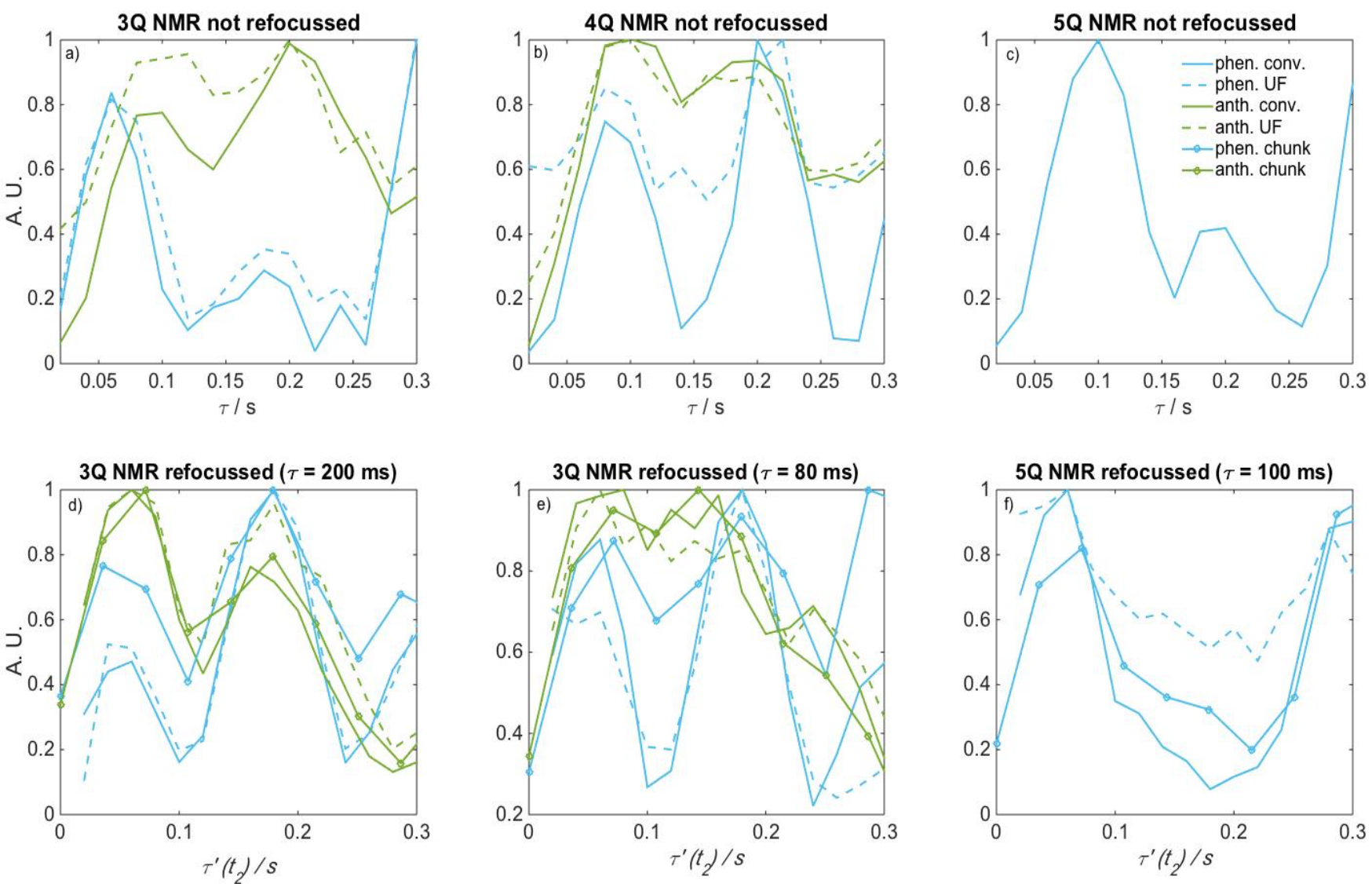

Fig. 5. (a-c), evolution of the peak volume (normalized to the highest peak volume observed in each trend) of phenol and naphthalene observed in conventional and UF $3 Q, 4 Q$ and $5 Q$ NMR spectra obtained using the sequence $A$, as function of the $\tau$ delay that was changed from 20 ms to 300 $\mathrm{ms}$. (d-f), evolution of the peak volume of the phenol and naphthalene in conventional and UF $3 Q, 4 Q$ and $5 Q$ NMR spectra, as function of the $\tau_{1}$ delay that was changed from $20 \mathrm{~ms}$ to $300 \mathrm{~ms}$, the length of the $\tau$ delay was kept fixed at the values shown in the title. Plot with the dotted lines represent data obtained from a FID (originally $800 \mathrm{~ms}$ long) truncated in nine chucks of $72 \mathrm{~ms}$.

The data obtained from conventional and UF experiments showed comparable maxima and trends. The plots in Figs. 5ac show three clear maxima for all the $M Q(s)$, in the range between $60 \mathrm{~ms}$ and $100 \mathrm{~ms}$, centred about $200 \mathrm{~ms}$ and at 300 ms. Choosing points corresponding to maxima (at $200 \mathrm{~ms}$ in the $3 Q$ experiments, at $80 \mathrm{~ms}$ in the $4 \mathrm{Q}$ experiments and at 100 $\mathrm{ms}$ in the $5 \mathrm{Q}$ experiments), a new set of experiments were performed using the sequence $B$ keeping $\tau$ fixed at these 
values and with $\tau_{1}$ changing from $20 \mathrm{~ms}$ to $300 \mathrm{~ms}$, as shown in Figs. $5 d-f$. From this data, we concluded that in order to acquire UF MQ spectra with of good quality, as that obtained in the conventional ones, build up times ranging between $80 \mathrm{~ms}-$ $200 \mathrm{~ms}$, and a delay (that can be introduced by a refocusing block or by a pre-acquisition delay) of about $60 \mathrm{~ms}-100 \mathrm{~ms}$ before the acquisition, have to be applied. $3 Q$ spectra showed in Fig. 4 (and Fig. 7 for the UF 4Q NMR experiments) were acquired using sequence $B$ with $\tau$ equal to $200 \mathrm{~ms}$ and $\tau_{1}$ equal to $180 \mathrm{~ms}, 4 \mathrm{Q}$ spectra were obtained with $\tau$ equal to $80 \mathrm{~ms}$ and $\tau_{1}$ equal to $60 \mathrm{~ms}, 5 \mathrm{Q}$ spectra were obtained with the $\tau$ delay equal to $100 \mathrm{~ms}$ and $\tau_{1}$ equal to $60 \mathrm{~ms}$, corresponding to maxima observed in plots showed in Fig. 5.

In order to illustrate the role of the refocusing delay, a complementary curve was obtained by processing data obtained with conventional MQ NMR pulse sequence (without spin-echo between mixing and acquisition) with an acquisition time $\left(t_{2}\right)$ of $800 \mathrm{~ms}$, but using a data chunk of $72 \mathrm{~ms}$ separated from the mixing pulse. The evolution of the peak volume obtained in the first nine chunks (Figs. 5d-f, dotted lines), matches that of the experiments using the refocusing delay. In this work the refocusing delay did not serve the purpose of pure-absorption processing of the data [43], which is in most cases not feasible for spatially encoded $2 \mathrm{D}$ spectra, but to add an additional time in the pulse sequence in order to allow the conversion of the anti-phase terms to in-phase term, increasing the intensity of the signal in the spectra.

\section{Sensitivity of UF MQ NMR}

While it provides a significant acceleration of multidimensional experiments, spatial encoding also comes with a sensitivity penalty $[38,44-45]$. Besides the reduction in signal due to the acquisition of a single scan, UF2DNMR experiments have reduced sensitivity per square root of measurement time, due to the increased noise level that comes with the use of a large acquisition bandwidth to cover the gradient-induced frequency dispersion. This effect can be characterised by comparing the signal-to-noise ratio (SNR) of, on the one hand, conventional experiments acquired with $N_{1}$ increments and a single scan per increment, and, on the other hand, UF experiments acquired with $N_{1}$ signal-averaged scans. SNR comparisons show that the UF version, as expected, has, in the direct dimension, a sensitivity penalty in the 5-25 range. The spread of SNR ratios is dues in part to the fact that the UF experiment is here implemented in constant time mode, which results in additional $\mathrm{J}$-modulation of peak intensities. Interestingly, the SNR ratio is less unfavourable for UF spectra in the indirect dimension, because of significant $t_{1}$ noise in the conventional experiments, while UF2DNMR spectra are essentially free of $t_{1}$ noise [44], SNR measurements for a selection of peaks is shown in Table S2.4-2.6.

\section{UF MQ NMR applied to reaction monitoring}

UF2DNMR has been shown to be useful to monitor chemical reaction at short reaction time and with increased time resolution [46]. UF MQ NMR could complement the set of available UF pulse sequences, especially for aromatic compounds. is illustrated here with the monitoring of a catalysed cycloaddition reaction with UF $4 Q$ NMR experiments. The reaction scheme of the studied inverse electron-demand aza-Diels-Alder reaction is shown in Fig. 6, which a variation of MaxQ from the reagents (MaxQ equal to 5 ) to the product a cyclohexene derivative with a very well-defined $4 Q$ arising from protons 8, 9, 10 and 11 .

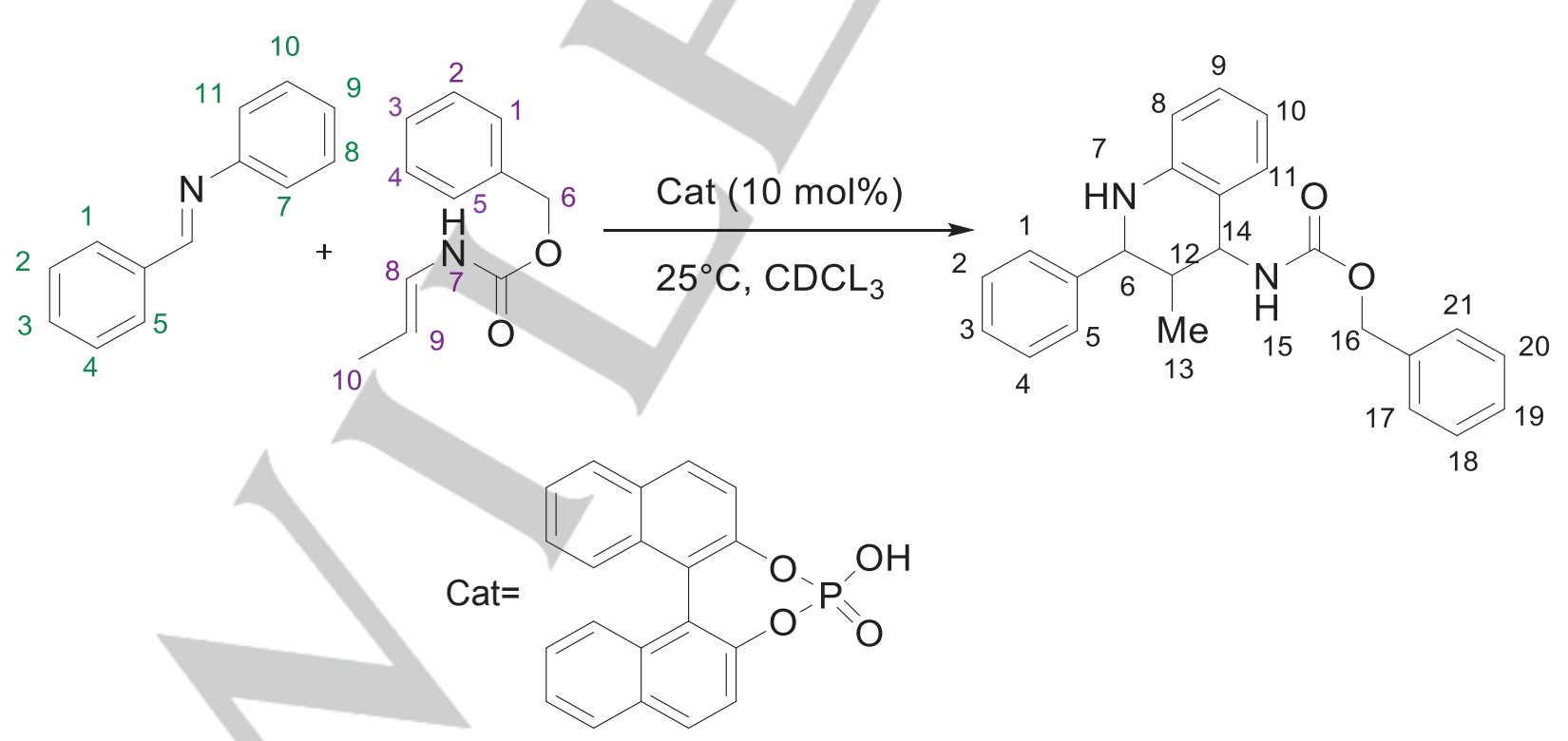

Fig. 6. Inverse electron-demand aza-Diels-Alder reaction catalysed by a racemic BINOL-phosphoric acid.

The 1D spectra of the initial reaction mixture and final cyclohexene derivative compound, as well as the assignment of the signals are shown in Fig. S3.1 and Table S3.1. Fig. 7 shows a selection of spectra from a time-series acquired during the reaction, which was carried out in an NMR tube, using either a room-temperature probe equipped with triple-axis gradients, or 
a cryoprobe equipped with a single-axis gradient. The $4 Q$ spectra of the diene (before the addition of the dienophile and the catalyst) and of the final mixture are also shown. The conventional $4 \mathrm{Q}$ NMR spectra of the reaction mixture and of the final compound are shown in Fig. S3.2.
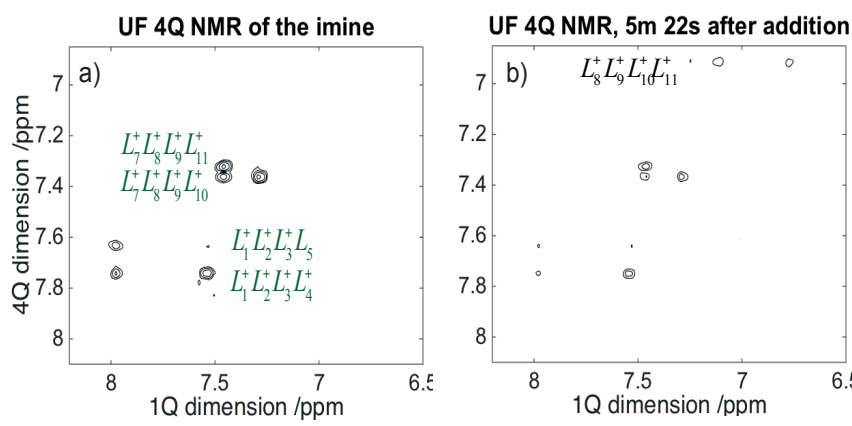

UF 4Q NMR, 7m 22s after addition
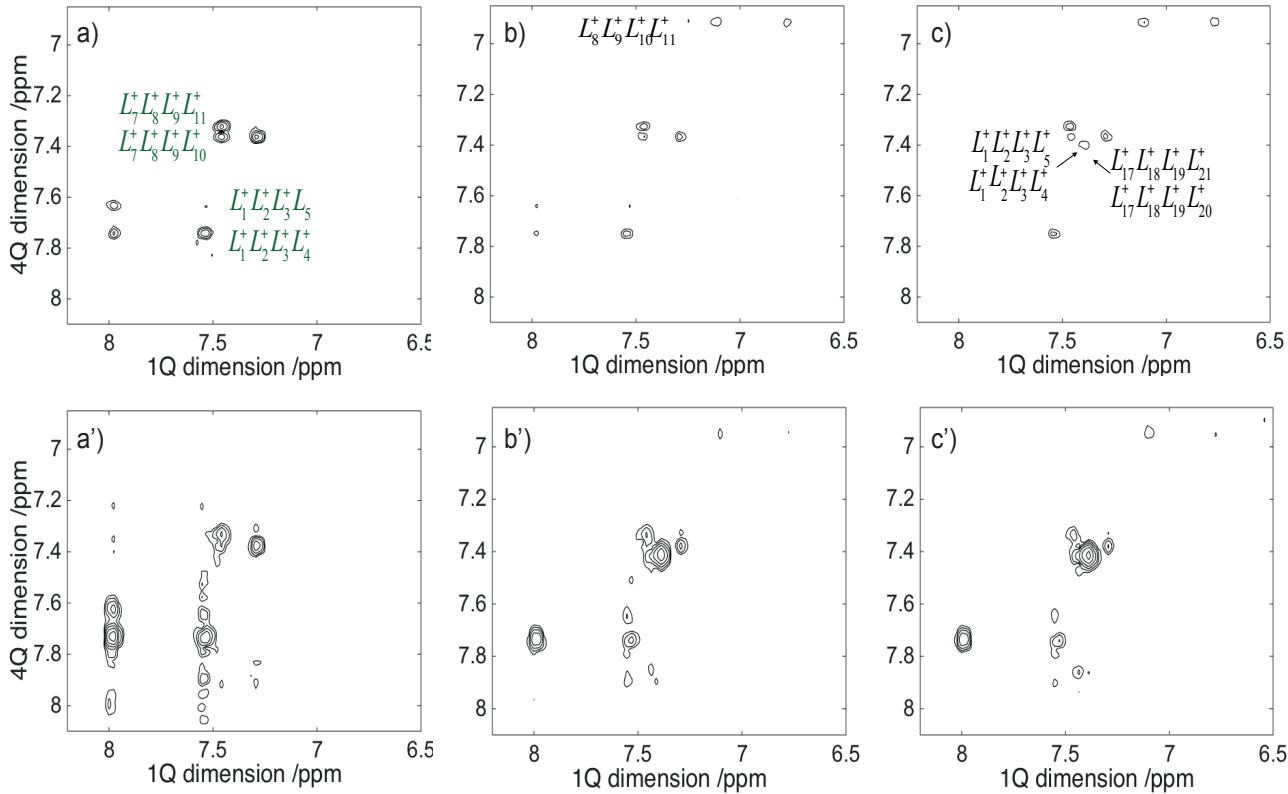
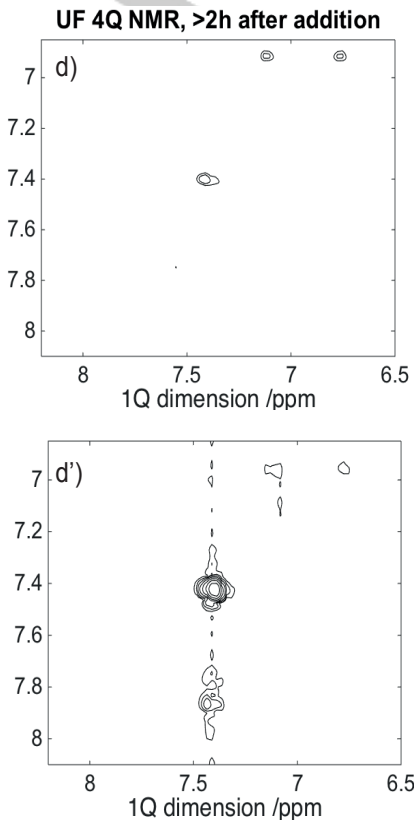

Fig. 7: UF 4Q NMR monitoring of a catalysed cycloaddition reaction, with (a-d) a room-temperature probe equipped with the triple-axis gradient probe, (a'-d') a cryoprobe with the single-axis gradient, UF 4 Q NMR spectra were acquired at different stage of the reaction. a) imine compound before the addition of the catalyst and the dienophile, b) reaction mixture 5 minutes and 22 seconds after the addition of the dienophile and the catalyst (4 minutes required for locking and shimming, plus the experimental time of 1 minute and 22 seconds), c) reaction mixture 7 minutes and 22 seconds after the addition of the dienophile and the catalyst, d) reaction mixture at the end of the reaction. The spectra were processed using contour levels at 3 times the rms of the noise, the colour code of the operators was established in according to chemical structure shown in Fig. 6. Spectra obtained with the triple-axis and single-axis probe had different spectra width in the UF dimension due to the difference in the amplitude of the encoding gradients, this figure is showing only the central region of the full spectra.

Signals belonging to the imine compound, the dienophile and the final cyclohexene compound can be assigned. In the first UF $4 Q$ NMR spectum (Fig. 7a), four intense signals arising from the $4 \mathrm{Q}$ of the imine compound $\left(L_{7}^{+} L_{8}^{+} L_{9}^{+} L_{11}^{+}\right.$at $7.32 \mathrm{ppm}$, $L_{7}^{+} L_{8}^{+} L_{9}^{+} L_{10}^{+}$at $7.36 \mathrm{ppm}, \quad L_{1}^{+} L_{2}^{+} L_{3}^{+} L_{5}^{+}$at $7.63 \mathrm{ppm}$ $L_{1}^{+} L_{2}^{+} L_{3}^{+} L_{4}^{+}$at $7.74 \mathrm{ppm}$ ), that gradually disappeared forming the final compound, characterized by one strong signal arising from the $4 \mathrm{Q}$ of the cyclohexene derivative $\left(L_{8}^{+} L_{9}^{+} L_{10}^{+} L_{11}^{+}\right.$at 6.92 ppm) and by four $4 \mathrm{Q}$ from the two phenyl groups $\left(L_{1}^{+} L_{2}^{+} L_{3}^{+} L_{5}^{+}\right.$, $L_{1}^{+} L_{2}^{+} L_{3}^{+} L_{4}^{+}, L_{17}^{+} L_{18}^{+} L_{19}^{+} L_{21}^{+}$and $L_{17}^{+} L_{18}^{+} L_{19}^{+} L_{20}^{+}$overlapped at $7.40 \mathrm{ppm}$ (at the same chemical shift of the signals of the dienophile) ;Fig. 6c-d).

Comparing the data obtained with two different probes highlights the relative merits of the two setups. As expected, the spectra acquired with a cryoprobe have a higher signal-to-noise ratio. For example, the $4 Q$ correlation of the two phenyl groups of the product, at $7.40 \mathrm{ppm}$, were already visible after 5 minutes after the addition of the dienophile and the catalyst, while they become visible after 7 minutes only in the experiments performed with the triple-axis gradient probe. On the other hand, the use of orthogonal gradients for coherence selection with the triple-axis gradient probe results in much cleaner spectra, so that a cryoprobe equipped with triple-axis gradients would be ideal for this type of experiments.

\section{Conclusions}

In summary, we have demonstrated the ultrafast acquisition of maximum quantum (MaxQ) spectra in a single scan, with performance comparable to those observed in the conventional experiments, and proposed an experimental strategy to determine the best experimental conditions with an optimal compromise between sensitivity and resolution, as well as a theoretical approach to quickly identify correlation peaks that can simplify the spectral analysis. The proposed UF MaxQ approach could be useful for the analysis of stable mixtures and for reaction monitoring that contain and /or involve structurally similar aromatic compounds.

\section{Acknowledgements}

This research was supported by the Agence Nationale de la Recherche (ANR-16- CE29-0012). 
Keywords: liquid sate NMR methods, small molecules, spin dynamics.

\section{References}

1. S. L. Robinette, R. Brüschweiler, F. C. Schroeder, A.S Edison, Acc Chem Res. 2012, 45(2), 288-97.

2. K. Bingol, D-. W. Li, B. Zhang, and R. Brüschweiler, Anal Chem. 2014, 86(11), 5494-501.

3. R. R. Ernst, G. Bodenhausen, A. Wokaun, Principles of Nuclear Magnetic Resonance in One and Two Dimensions, Clarendon Press, Oxford, 1991.

4. M. Munowitz and A. Pines, Adv. Chem. Phys. 1987, 66, 1152.

5. M. P. Munowitz and A. Pines, Science. 1986, 233, 525.

6. T. J. Norwood, Prog. Nucl. Magn. Reson. Spectrosc. 1992, 24 , $295-375$

7. T. J. Norwood, Multiple quantum spectroscopy of liquid samples, in D. M. Grant, R.K. Harris (Eds.), Encyclopedia of Magnetic Resonance, John Wiley and Sons Chichester, 2007, pp. 3181-3188.

8. S. Sinton and A. Pines, Chem. Phys. Lett. 1980, 76, 263-267.

9. G. N. Manjunatha Reddy and S. Caldarelli, Anal Chem. 2010, 82(8), 3266-9.

10. G. N. Manjunatha Reddy and S. Caldarelli, Magn Reson Chem. 2013, 51(4), 240-4.

11. M. Piotto, G. N. Manjunatha Reddy and S. Caldarelli, J Magn Reson. 2011, 213(1), 107-11.

12. G. N. Reddy, and S. Caldarelli, Chem Commun (Camb). 2011, 47(14), 4297-9.

13. C. E. Hughes, Prog. Nucl. Magn. Reson. Spectrosc. 2004, $45,301-313$

14. A. Lesage, C. Auger, S. Caldarelli, and L. Emsley, Am. Chem. Soc. 1997, 119, 7867.

15. A. Bax, R. Freeman, and Stewart P. Kempsell, J. Am. Chem. Soc. 1980, 102 (14), 4849-4851.

16. M. H. Levitt and R. R. Ernst, Chem. Phys. Lett. 1983, 100, 119.

17. L. Rouger, B. Gouilleux, P. Giraudeau, Fast n-Dimensional Data Acquisition Methods in: J. C. Lindon, G.E. Tranter, D. W. Koppenaal (Eds.), Encyclopedia of Spectroscopy and Spectrometry, 3rd edn. Academic Press, Oxford, 2017, pp. 588-596.

18. P. Schanda and B. Brutscher, J Am Chem Soc. 2005, 127(22), 8014-5.

19. A.S. Stern, K-.B. Li, J. C. Hoch, J Am Chem Soc. 2002, 124, 1982-93

20. H. Barkhuijsen R. de Beer, W. M. M. Bovee, D. van Ormondt, J. Magn. Reson. 1985, 61, 465-481.

21. J. C. Hoch, J. Magn. Reson. 1985, 64(3), 436-440.
22. E. Kupce, and R. Freeman, J Magn Reson. 2003, 162, 30010.

23. Kupce, E. and R. Freeman, J Magn Reson. 2003, 162(1), 158-65.

24. C. D. Eads, and I. Noda, J Am Chem Soc. 2002, 124(6), 1111-8.

25. O. Cloarec, M. E. Dumas, A. Craig, R. H. Barton, J. Trygg, J. Hudson, et al., Anal Chem. 2005, 77(5), 1282-9.

26. I. E. Ndukwe, A. Shchukina, K. Kazimierczuk and C. P. Butts, Chem. Commun. 2016, 52(86), 12769-12772.

27. Y. Wu, C. D., Agostino, D. J. Holland, L. F. Gladden, Chem. Commun. 2014, 50, 14137-14140.

28. R. Dass, W. Kozminski, K. Kazimierczuk. Anal. Chem. 2015 , $87,1337-1343$

29. M. Mayzel, J. Rosenlöw, L. Isaksson, V. Y.Orekhov, J. Biomol. NMR. 2014, 58, 129-39.

30. P. Giraudeau, and L. Frydman, Annu Rev Anal Chem (Palo Alto Calif). 2014, 7, 129-61.

31. M. Gal, M. Mishkovsky, and L. Frydman, J. Am. Chem. Soc. 2006, 128 (3), 951-956.

32. B. Gouilleux, B. Charrier, E. Danieli, J-. N. Dumez, S. Akoka, F.X. Felpi, M. Rodriguez-Zubiri M, P. Giraudeau, Analyst. 2015, 140, 7854-8.

33. R. Boisseau, U. Bussy, P. Giraudeau and M. Boujtita, Anal. Chem. 2015, 87(1), 372-375.

34. A.L.Guennec, P. Giraudeau S. Caldarelli, J-.N.Dumez, Chem Commun (Camb). 2015, 51(2), 354-7.

35. L. Rouger, B. Gouilleux, M. Pourchet-Gellez, J-. N.Dumez, P. Giraudeau, Analyst. 2016, 141(5), 1686-92.

36. L. Frydman, T. Scherf, A. Lupulescu, Proc Natl Acad Sci US A. 2002, 99(25), 15858-62.

37. M. Mishkovsky and L. Frydman, Annu Rev Phys Chem. 2009, 60, 429-48.

38. A. Tal and L. Frydman, Prog Nucl Magn Reson Spectrosc. 2010, 57(3), 241-92.

39. L. Frydman, A. Lupulescu, T. Scherf, J Am Chem Soc. 2003, 125(30), 9204-17.

40. I. Kuprov, J Magn Reson. 2016, 270, 124-135.

41. H. J. Hogben, M. Krzystyniak, G. T. Charnock, P. J. Hore, I. Kuprov, J Magn Reson. 2011, 208(2), 179-94.

42. (a) G. Dagousset, J. Zhu, G. Masson, J. Am. Chem. Soc. 2011, 133, 14804-13; b) G. Bernadat, G. Masson, Synlett. 2014, 25, 2842-67.

43. A. J.Pell and J. Keeler, J Magn Reson. 2007, 189(2), 293-9.

44. M. Pathan, S. Akoka, I. Tea, B. Charriera and P. Giraudeau, Analyst. 2011, 136, 3157.

45. J-. N. Dumez, Prog. Nucl. Magn. Reson. 2018, 109, 101-1.

46. A. Herrera , E. Fernández-Valle, R. Martínez-Álvarez, D. Molero-Vílchez, Z. D. Pardo-Botero, E. Sáez-Barajas, Magn. Reson. Chem. 2015, 53, 952-70. 


\section{ARTICLE}

Text for Table of Contents

Maria Grazia Concilio, Corentin

Jacquemmoz, Dina Boyarskaya,

Géraldine Masson and Jean-Nicolas

Dumez*

Page No. 1 - Page No. 9

Ultrafast maximum quantum NMR spectroscopy for the analysis of aromatic mixtures
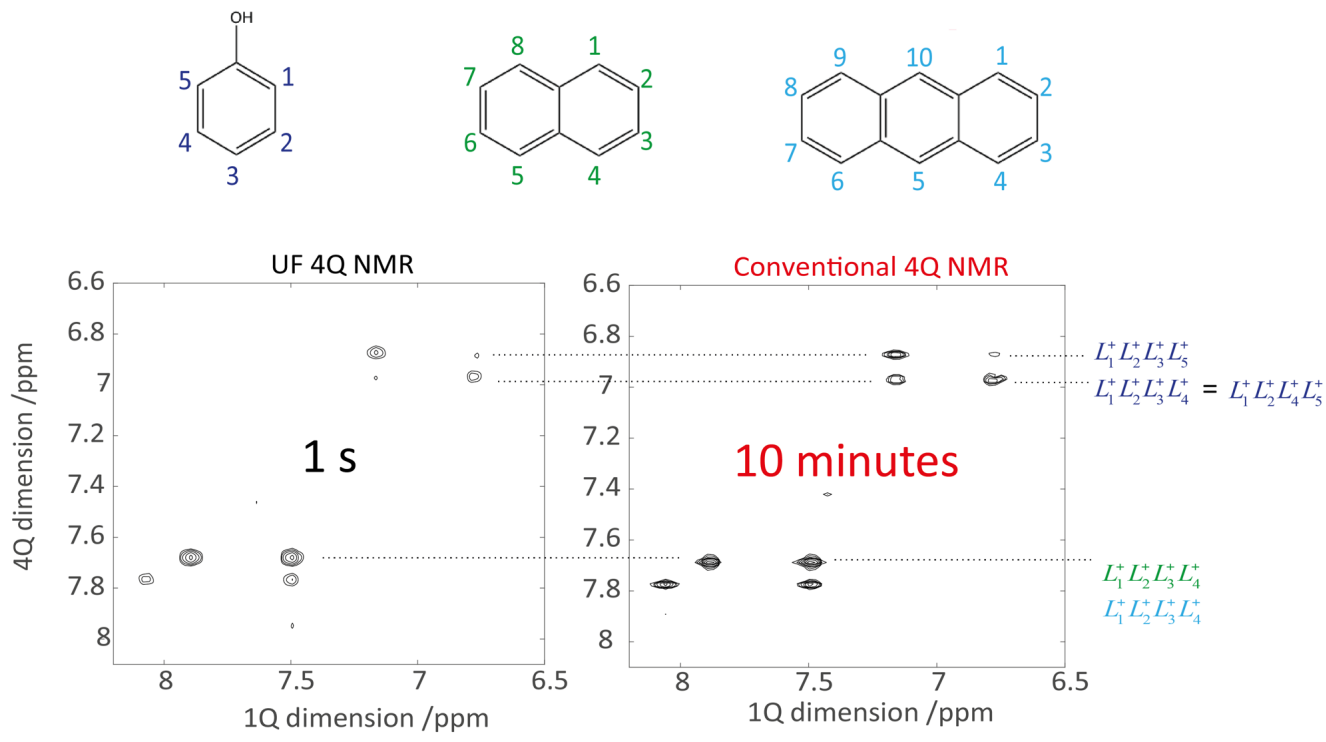

Ultrafast acquisition of multiple quantum spectra of an aromatic mixture in one second 